\title{
Relationship between foot function and medial knee joint loading in people with medial compartment knee osteoarthritis
}

\author{
Pazit Levinger ${ }^{1,2 *}$, Hylton B Menz ${ }^{2}$, Adam D Morrow ${ }^{2}$, John R Bartlett ${ }^{3}$, Julian A Feller ${ }^{2}$ and Neil R Bergman ${ }^{3}$
}

\begin{abstract}
Background: Dynamic joint loading, particularly the external knee adduction moment (KAM), is an important surrogate measure for the medio-lateral distribution of force across the knee joint in people with knee osteoarthritis (OA). Foot motion may alter the load on the medial tibiofemoral joint and hence affect the KAM. Therefore, this study aimed to investigate the relationship between tibia, rearfoot and forefoot motion in the frontal and transverse planes and the KAM in people with medial compartment knee OA.

Method: Motion of the knee, tibia, rearfoot and forefoot and knee moments were evaluated in 32 patients with clinically and radiographically-confirmed OA, predominantly in the medial compartment. Pearson's correlation coefficient was used to investigate the association between peak values of tibia, rearfoot and forefoot motion in the frontal and transverse planes and $1^{\text {st }}$ peak KAM, $2^{\text {nd }}$ peak KAM, and the knee adduction angular impulse (KAAl).

Results: Lateral tilt of the tibia was significantly associated with increased $1^{\text {st }}$ peak KAM $(r=0.60, p<0.001), 2^{\text {nd }}$ peak KAM $(r=0.67, p=0.001)$ and KAAI $(r=0.82, p=0.001)$. Increased peak rearfoot eversion was significantly correlated with decreased $2^{\text {nd }}$ peak KAM $(r=0.59, p<0.001)$ and KAAI $(r=0.50, p=0.004)$. Decreased rearfoot internal rotation was significantly associated with increased $2^{\text {nd }}$ peak KAM $(r=-0.44, p=0.01)$ and KAAI $(r=-0.38, p=0.02)$, while decreased rearfoot internal rotation relative to the tibia was significantly associated with increased $2^{\text {nd }}$ peak KAM $(r=0.43, p=0.01)$. Significant negative correlations were found between peak forefoot eversion relative to the rearfoot and $2^{\text {nd }}$ peak KAM $(r=-0.53, p=0.002)$ and KAAI $(r=-0.51, p=0.003)$ and between peak forefoot inversion and $2^{\text {nd }}$ peak KAM $(r=-0.54$, $p=0.001)$ and KAAI $(r=-0.48, p=0.005)$.

Conclusion: Increased rearfoot eversion, rearfoot internal rotation and forefoot inversion are associated with reduced knee adduction moments during the stance phase of gait, suggesting that medial knee joint loading is reduced in people with $O A$ who walk with greater foot pronation. These findings have implications for the design of load-modifying interventions in people with knee OA.
\end{abstract}

Keywords: Knee osteoarthritis, Foot motion, Knee adduction moment

\section{Background}

Knee osteoarthritis $(\mathrm{OA})$ is a chronic debilitating condition, affecting a substantial number of older people worldwide [1,2]. People with knee OA suffer from pain and difficulties in performing activities of daily living. OA in the medial compartment of the knee is highly prevalent

\footnotetext{
* Correspondence: pazit.levinger@vu.edu.au

'Institute of Sport, Exercise and Active Living, College of Sport and Exercise

Science, Victoria University, Melbourne Vic 8001, Australia

2Lower Extremity and Gait Studies Program, Faculty of Health Sciences, La

Trobe University, Melbourne Vic 3086, Australia

Full list of author information is available at the end of the article
}

and has been attributed to the increased load transmitted across the medial compartment of the knee joint [3]. Although several factors have been associated with the incidence and progression of OA, particularly medial compartment knee OA, the aetiology of knee OA is not fully understood. Biomechanical factors associated with joint loading have been the focus of recent studies as an important element in the pathogenesis of knee OA.

Dynamic joint loading, particularly the external knee adduction moment (KAM), has received attention as an important surrogate measure of the medio-lateral distribution 
of force across the knee joint. Although the evidence for the contribution of KAM to the development of knee OA is inconsistent [4], several studies have shown increased KAM to be associated with knee OA severity and varus malalignment [5]. Consequently, several treatment strategies, including load modifying interventions, have been suggested to reduce the load on the medial compartment of the knee by altering the KAM [6-12].

The KAM is influenced by variation in lower limb alignment and motion during gait [13-17]. Varus limb alignment, which is commonly observed in people with medial compartment knee OA, has been shown to increase the incidence and progression of knee OA [18-20]. Recent studies have also reported that people with medial compartment knee OA have a relatively pronated foot posture [21-23] and demonstrate foot kinematic patterns that are indicative of a less mobile, everted foot type [24] compared to controls. Moreover, the degree of varus alignment may also affect foot motion during walking which may lead to a compensatory response to allow typical function of the foot during ambulation [24]. Footwear and orthotic interventions, therefore, have been studied as a method for altering medial knee loading by altering foot motion [6-12].

The mechanism by which footwear and orthotic interventions aim to reduce the knee adduction moment is by pronating the foot through lateral inclination of the insole (thereby laterally shifting the centre of pressure) [25]. However, it is unclear if variation in foot motion, particularly foot eversion, influences the KAM. In order to better understand how knee joint loading is influenced by lower limb motion, this study investigated the relationship between tibia, rearfoot and forefoot motion in the frontal and transverse planes and KAM in people with medial compartment knee OA. We hypothesised that kinematic parameters indicative of greater foot pronation (internal tibial rotation, frontal plane rearfoot eversion and frontal plane forefoot inversion) would be associated with a reduction in medial knee joint loading.

\section{Methods}

This project was part of a larger study that investigated gait (swing phase mechanics, particularly minimum foot clearance), balance and falls risk in people before and after knee arthroplasty. A power calculation to determine the sample size, therefore, was based on minimum foot clearance parameters. Data from a previous study [26] which investigated the toe clearance of elderly fallers and non-fallers were used to determine the number of participants required. A sample size calculation indicated that for $80 \%$ power and a $p$ value of 0.05 at least 25 participants were required. To mitigate the possible effect of subject drop out for the surgical group, a total of 32 participants were considered to be sufficient. Thirty two participants (16 females, average age $65.8 \pm 7.5 \mathrm{yr}$, height $168.8 \pm 9.5$ and body weight $85.1 \pm 13.6 \mathrm{~kg}$ ) with diagnosed OA predominantly in the medial compartment of the knee, determined by radiographic assessment [24], participated in the study. Detail of the foot posture of the participants has previously been reported [21]. The severity of knee OA was based on the loss of joint space determined by an orthopaedic surgeon from radiographic images [27] and was graded as follows: 1- less than a half of joint space loss (mild), 2 - more than a half of joint space loss; bone on bone (moderate) and 3 - bone deformity/loss of bone (severe). Each compartment of the knee joint (medial compartment, lateral compartment and patellofemoral compartment) was graded and participants with predominantly medial compartment knee OA (severity grade 2-3) were included in the study. Sixteen participants had moderate severity of OA (grade 2) and 16 participants had severe OA (grade 3) based on radiographic assessment [27]. Participants were included if they were able to walk independently and were excluded if they had uncontrolled systemic disease and or a preexisting neurological or other orthopaedic condition that affected their walking. Participants were recruited from the La Trobe University Medical Centre, the Warringal Private Medical Centre and through advertisements in local newspapers. Ethics approval was obtained from the Faculty of Health Sciences Human Ethics Committee, La Trobe University. All participants were informed about the nature of the study and signed a consent form prior to participation.

\section{Procedure}

\section{Instrumentation}

A three dimensional motion analysis system (Vicon MX, Vicon Motion System Ltd, Oxford, England) with 10 cameras (8 MX3 and 2 MX40) was used to capture and analyse motion of the lower leg with a sampling frequency of $100 \mathrm{~Hz}$. Two force plates (Kistler, type 9865B, Winterthur, Switzerland and AMTI, Watertown, MA, USA) $(1000 \mathrm{~Hz})$ were used to capture ground reaction forces and identify gait cycle events. The marker trajectories and force platform data were captured synchronously using the Vicon Nexus software package. The force plate data were then re-sampled at $100 \mathrm{~Hz}$ for the calculation of knee joint moments.

\section{Kinematic evaluation}

Participants were required to attend a single testing session at the gait laboratory at La Trobe University. Lower leg and foot motion of the symptomatic leg (or the most symptomatic leg in a case of bilateral involvement) was assessed. To assess the three dimensional motion of the lower limb including, knee, tibia, rearfoot and forefoot and knee moments in the frontal plane, retro-reflective markers were attached on anatomical landmarks over 
the lower legs in accordance with the Oxford Foot Model (OFM) marker set and Plug In Gait (PIG) [28] as described by Stebbins et al. [29]. Retro-reflective markers were then placed over the anatomical land marks on the pelvis, thigh, tibia, rearfoot and forefoot as described in details in Levinger et al. [24]. The OFM modelled the tibia, rearfoot and forefoot as rigid segments. The tibial segment was comprised of markers placed on the medial malleolus, the lateral malleolus, the anterior aspect of the tibial crest, the tibial tuberosity and the head of the fibula. The rearfoot segment was defined by placing markers on the sustentaculum tali, the lateral calcaneus, the heel (distal part of the calcaneus), the posterior proximal calcaneus and a peg marker was placed on the posterior calcaneus between the heel and proximal calcaneus markers. The forefoot segment was defined by placing markers on the most distal, medial aspect of the first metatarsal shaft, the most proximal and distal lateral aspects of the fifth metatarsal shaft, and midway between the second and third metatarsal heads.

Prior to kinematic evaluation of the lower leg motion, a relaxed standing calibration trial was captured with knee alignment devices (KAD, Motion Lab Systems Inc. LA, USA). Several markers, used only in the static trials (medial malleoli, proximal heel, and first metatarsal), were removed prior to the dynamic trials as described in Stebbins et al. [29]. The locations of the joints centre were calculated from PIG [28]. Moreover, the location of the knee joint centre, calculated from PIG, was further used in the OFM for the tibia segment definition.

Participants were asked to walk at a comfortable walking pace along a $12 \mathrm{~m}$ walkway and five successful trials were collected for each leg. A successful trial was defined when the participant's foot landed on the centre of the force plate without any interference to their gait. For each trial, gait events were detected using vertical ground reaction force data to determine initial foot contact and toe off. Multiple trials were practiced until participants were comfortable and walking with consistent velocity. The peak values of interest (maximum value during the stance phase) of each trial were extracted separately; the average of the five trials was then used in the analysis. All gait variables of interest generated by the model were normalised to the gait cycle and timing of peak angular variables were then expressed as a percentage of the gait cycle.

The magnitude of peak angular motion of the tibia, rearfoot relative to the global coordinate system (laboratory), rearfoot relative to the tibia and forefoot relative to the rearfoot in the frontal and transverse planes during the gait cycle were extracted including the following angles: (i) tibia lateral tilt and internal/external rotations (ii) peak rearfoot eversion/inversion and internal/external rotation relative to the tibia; (iii) rearfoot eversion/inversion and internal/external rotation relative to the global reference system (laboratory); (iv) peak forefoot abduction/ adduction; and eversion/inversion. Knee frontal plane angular motion (knee varus) during initial contact and during stance (peak knee varus) and external KAM (normalised to $\%$ of body weight"height) including $1^{\text {st }}$ peak and $2^{\text {nd }}$ peak were also extracted. Knee adduction angular impulse (KAAI - the integral of the frontal plane knee moment over the stance phase of the gait cycle) [30] was also calculated. Figure 1 depicts the influence of knee alignment on the KAM, and Figure 2 depicts the three kinetic variables extracted from this data $\left(1^{\text {st }}\right.$ peak KAM, $2^{\text {nd }}$ peak KAM and KAAI).

\section{Knee pain, function and stiffness}

Clinical severity of OA including physical function, pain and stiffness were assessed using the Western Ontario and McMaster University Osteoarthritis Index (WOMAC) [31]. This index, using $10 \mathrm{~mm}$ visual analogue scale, assesses the severity of the knee pain during 5 daily activities (range $0-500$ ), stiffness (range $0-200$ ), and the severity of impairment of lower-extremity function during 17 activities $(0-1700)$. A score of zero represents no pain or difficulty with physical function and higher scores represent worse functional health. All three subcategories are summed to give a global WOMAC score (range 0 - 2400).

\section{Statistical analysis}

Pearson's correlation coefficient was used to investigate the relationship between peak KAMs $\left(1^{\text {st }}\right.$ peak KAM and $2^{\text {nd }}$ peak KAM), KAAI and the following parameters: peak values of tibia, rearfoot (both relative to the laboratory and relative to the tibia) and forefoot motion in the frontal and transverse planes.

\section{Results}

Mean \pm standard deviation external KAMs (\% bodyweight " height) were as follows: $1^{\text {st }}$ peak KAM $=3.3 \pm 1.6,2^{\text {nd }}$ peak $\mathrm{KAM}=2.8 \pm 1.1$ and $\mathrm{KAAI}=1.26 \pm 0.5$. Correlations between foot motion and KAM-related variables are shown in Table 1. Angular motion of the tibia, rearfoot and forefoot in the frontal and transverse planes are presented in Figures 3 and 4 . The knee OA group reported mild pain of $171.8 \pm 99.9$, function $502.5 \pm 330.9$ and stiffness $83.7 \pm 49.8$ with WOMAC total score of $758.1 \pm 447.1$.

Greater lateral tilt of the tibia was significantly correlated with increased KAMs and KAAI $(r=0.60$ to $0.82, p=0.001$ ). No correlations were found between tibial rotation and KAMs (Table 1). Significant positive correlations were found between peak rearfoot eversion relative to the laboratory and $2^{\text {nd }}$ peak KAM $(r=0.59, p<0.001)$ and KAAI $(r=0.50, p=0.004)$, indicating decreased adduction 


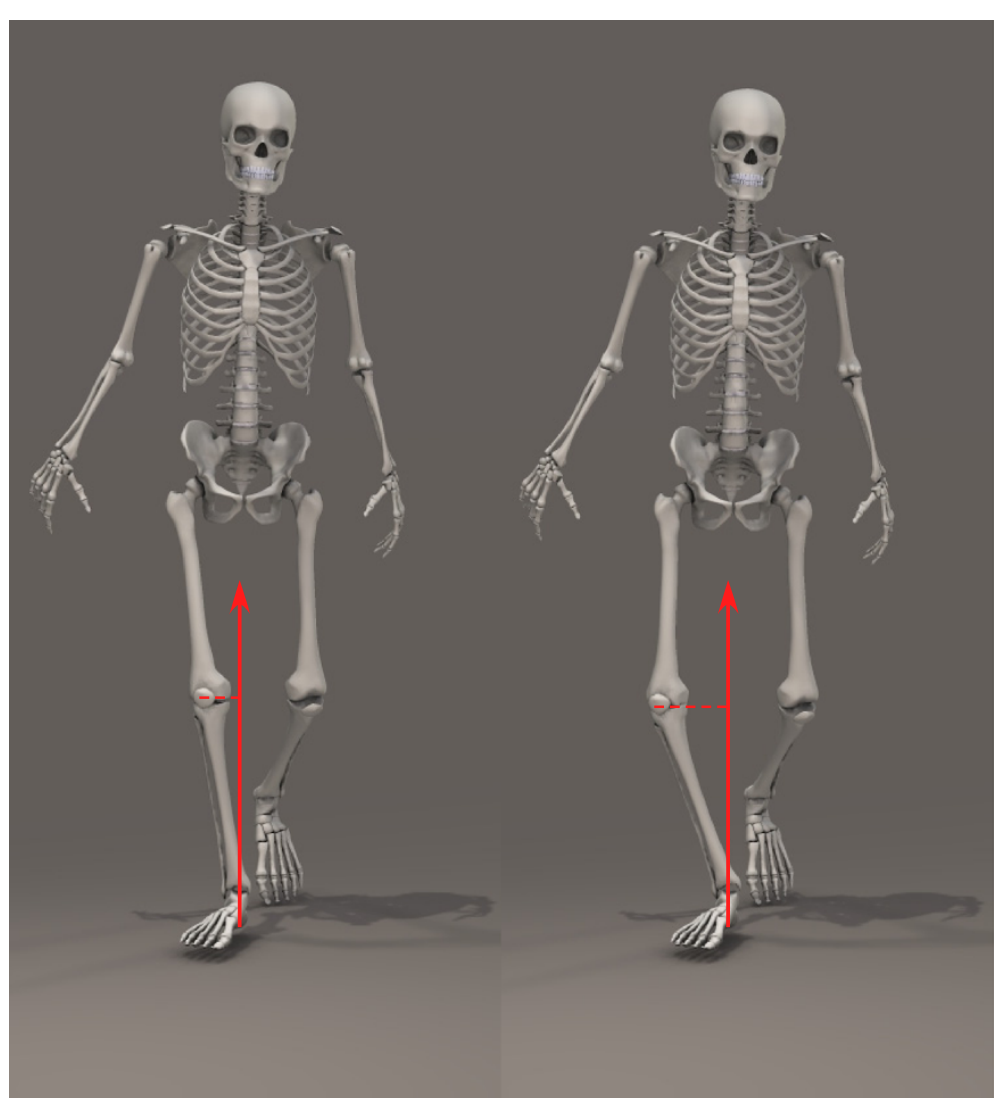

Figure 1 The knee adduction moment (KAM) increases when walking with greater varus alignment of the knee (shown on the right) as the perpendicular distance of the ground reaction force vector from the knee joint centre is greater, resulting in a longer moment arm.

moments with greater rearfoot eversion. Reduced rearfoot internal rotation relative to the laboratory was significantly correlated with greater $2^{\text {nd }}$ peak KAM $(r=-0.44, p=0.01)$ and KAAI $(r=-0.38, p=0.02)$. Similarly, increased rearfoot external rotation relative to the laboratory was significantly correlated with greater $2^{\text {nd }}$ peak KAM $(r=-0.54, p=0.001)$ and KAAI $(r=-0.48$, $p=0.005)$. No significant correlations were found between either peak rearfoot eversion or inversion relative to the tibia and any of the KAMs. Reduced rearfoot internal

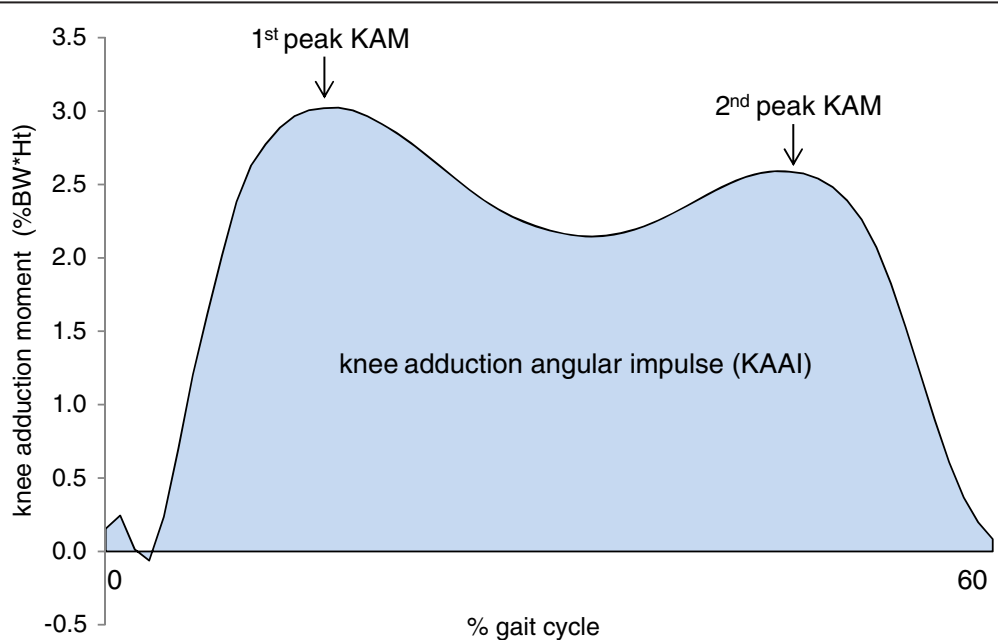

Figure 2 Frontal plane external KAMs: $1^{\text {st }}$ peak KAM, $2^{\text {nd }}$ peak KAM and knee adduction angular impulse (KAAI), which represents the area under the curve. 
Table 1 Correlations between peak rearfoot and forefoot motion in the frontal plane and peak knee adduction moments (KAM) and knee adduction angular impulse (KAAI)

\begin{tabular}{|c|c|c|c|c|c|c|}
\hline \multirow[b]{2}{*}{ Parameters } & \multicolumn{2}{|c|}{$1^{\text {st }}$ peak KAM } & \multicolumn{2}{|c|}{$2^{\text {nd }}$ peak KAM } & \multicolumn{2}{|l|}{ KAAI } \\
\hline & $r$ value & $p$ value & $r$ value & $p$ value & $r$ value & $p$ value \\
\hline \multicolumn{7}{|c|}{ Tibia relative to laboratory } \\
\hline Lateral tilt & $0.60^{*}$ & $<0.001$ & $0.67^{*}$ & 0.001 & $0.82^{*}$ & $<0.001$ \\
\hline Internal Rotation & 0.27 & 0.13 & 0.17 & 0.34 & 0.19 & 0.28 \\
\hline External Rotation & 0.14 & 0.44 & 0.19 & 0.29 & 0.14 & 0.43 \\
\hline \multicolumn{7}{|c|}{ Rearfoot relative to the tibia } \\
\hline Peak eversion & -0.12 & 0.48 & 0.25 & 0.16 & 0.07 & 0.69 \\
\hline Peak inversion & -0.08 & 0.63 & 0.10 & 0.56 & 0.03 & 0.85 \\
\hline Internal Rotation & -0.02 & 0.89 & $0.43^{*}$ & 0.01 & 0.29 & 0.10 \\
\hline External Rotation & -0.22 & 0.20 & 0.28 & 0.10 & 0.07 & 0.67 \\
\hline \multicolumn{7}{|c|}{ Rearfoot relative to laboratory } \\
\hline Peak eversion & 0.21 & 0.24 & $0.59^{*}$ & $<0.001$ & $0.50^{*}$ & 0.004 \\
\hline Peak inversion & 0.07 & 0.69 & 0.33 & 0.06 & 0.33 & 0.05 \\
\hline Internal Rotation & -0.18 & 0.30 & $-0.44^{*}$ & 0.01 & $-0.38^{*}$ & 0.02 \\
\hline External Rotation & -0.22 & 0.20 & $-0.54^{*}$ & 0.001 & $-0.48^{*}$ & 0.005 \\
\hline \multicolumn{7}{|c|}{ Forefoot relative to rearfoot } \\
\hline Peak eversion & -0.26 & 0.13 & $-0.53^{*}$ & 0.002 & $-0.51^{*}$ & 0.003 \\
\hline Peak inversion & -0.28 & 0.11 & $-0.54^{*}$ & 0.001 & $-0.48^{*}$ & 0.005 \\
\hline Abduction & 0.18 & 0.30 & 0.14 & 0.42 & 0.13 & 0.47 \\
\hline Adduction & 0.19 & 0.28 & 0.13 & 0.47 & 0.14 & 0.42 \\
\hline
\end{tabular}

Note: A positive correlation indicates increased peak rearfoot eversion and decreased rearfoot inversion is associated with decreased adduction moments. A negative correlation indicates reduced rearfoot internal rotation and increased external rotation relative to the laboratory is associated with greater $2^{\text {nd }}$ peak KAM and KAAl.

* significant at $p<0.05$.

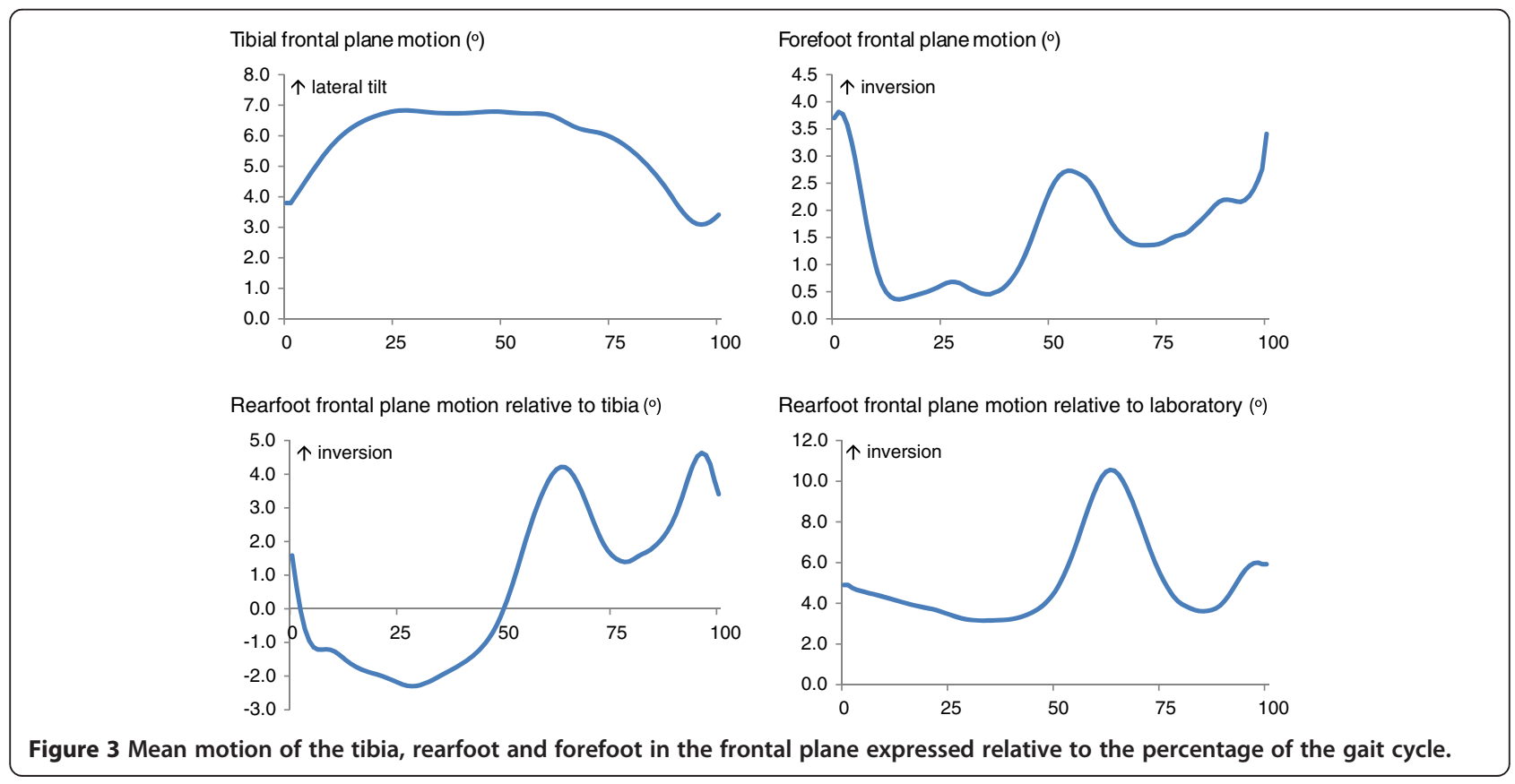



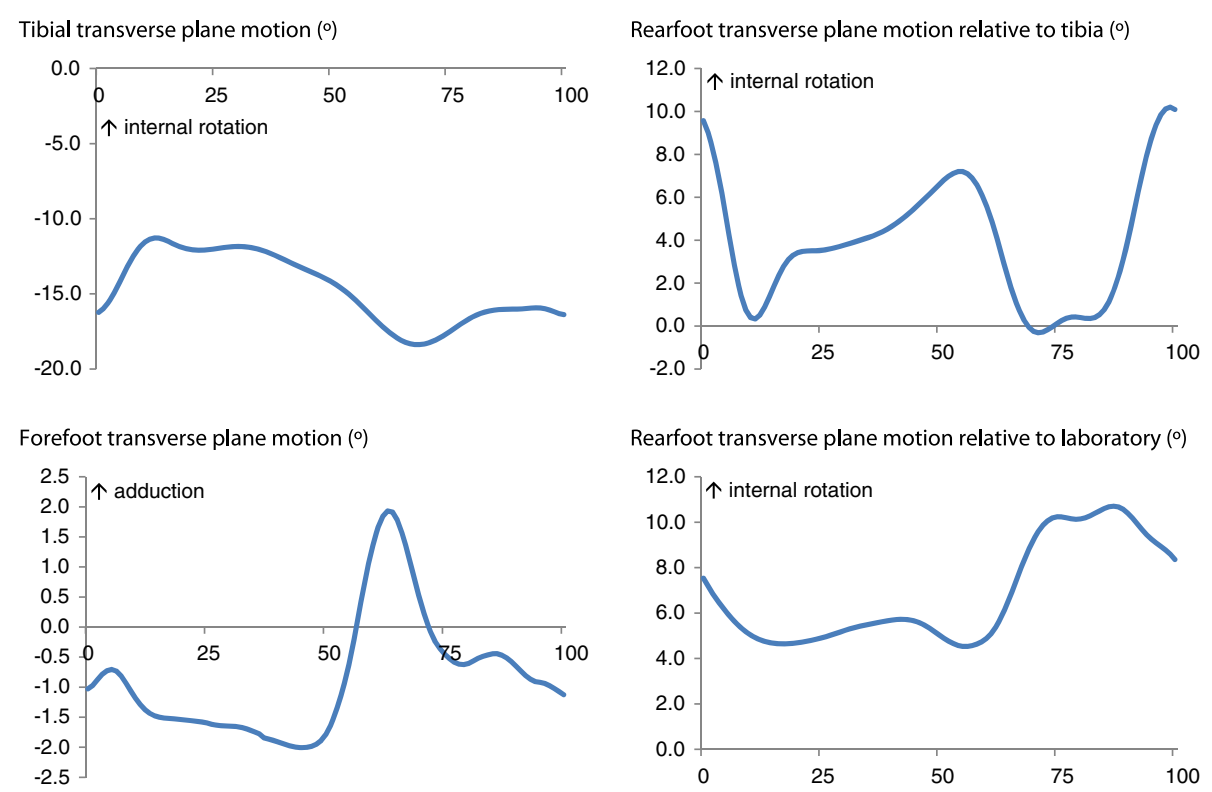

Figure 4 Mean motion of the tibia, rearfoot and forefoot in the transverse plane expressed relative to the percentage of the gait cycle.

rotation relative to the tibia was significantly correlated with increased $2^{\text {nd }}$ peak KAM $(r=0.43, p=0.01)$.

Significant negative correlations were found between peak forefoot eversion and $2^{\text {nd }}$ peak KAM $(r-0.53$, $p=0.002)$ and between peak forefoot inversion and $2^{\text {nd }}$ peak KAM $(r=-0.54, p=0.001)$. Similar correlations were also found between forefoot peak eversion and inversion and KAAI. The forefoot is generally inverted during the stance phase as it is affected by rearfoot eversion [24]. Therefore, a negative correlation indicates an association between increased forefoot inversion and reduced knee impulse and KAMs.

\section{Discussion}

Load-modifying interventions have been proposed as a strategy to reduce medial compartment knee loading (by reducing the external KAM) in people with knee OA, however equivocal findings have been reported regarding their effectiveness $[10,25,32,33]$. These strategies rely on altering knee joint loading by modifying movement of the foot, suggesting that by influencing foot motion, the moment arm of the ground reaction force that passes medially to the knee joint centre is reduced (Figure 1). Understanding the relationship between lower leg and foot motion and KAMs can therefore provide useful information to help optimise intervention strategies. In the present study, we found several associations between foot and tibia motion and external adduction moments at the knee which may influence the design of loadaltering interventions for knee OA.

Knee varus is frequently observed in people with medial compartment knee OA, with evidence suggesting that knee varus alignment increases the incidence and progression of OA [18-20]. We found significant correlations between lateral tibial tilt and KAMs and KAAI, indicating that greater tibial tilt increases the load on the medial compartment of the knee joint. These findings were expected, as greater tibial lateral tilt would increase the perpendicular distance of the ground reaction force vector from the knee joint centre, resulting in a greater moment arm and adduction moment (Figure 1). No correlation, however, was found in the transverse plane. Interestingly, an association between OA progression and torsional deformity in the tibia has previously been reported, as a decrease in tibial external rotation was accompanied by an increase in disease severity [34]. We have previously reported that people with knee OA have greater tibial internal rotation compared to aged-matched controls [35], and a reduction in tibial internal rotation was observed in patients who underwent realignment of the knee following knee replacement surgery [35]. Furthermore, internal torsion and varus deformity have been associated with increased loads on the medial compartment of the knee [36]. The lack of correlation in the present study between tibia rotation and KAMs may be related to a possible restriction in tibial motion as patients may be "pushed" to the end range of motion due to years of walking in the same pattern. However, due to the cross-sectional design of these studies, we are unable to infer the direction of causation between tibial rotation and the load on the medial compartment of the knee.

A significant association between rearfoot eversion relative to the laboratory and KAMs and KAAI was 
found, indicating that increased foot pronation is associated with reduced medial knee joint loading. In our previous work, the same knee OA group exhibited a relatively pronated foot type compared to an age-matched control group [21]. Greater internal rotation and reduced external rotation of the rearfoot relative to the lab were also associated with reduced $2^{\text {nd }}$ peak KAM and KAAI. Movement of the rearfoot in the frontal plane is coupled to internal rotation of the rearfoot, as previously reported in flat archedfeet [37]. Consequently, greater rearfoot eversion and internal rotation were associated with reduction of the overall medial loading during the stance phase of gait. Unlike rearfoot motion relative to the tibia, rearfoot motion relative to the laboratory is an independent measure of absolute rearfoot motion which may therefore more closely represent the link between rearfoot motion and knee moments.

Significant correlations were also found between forefoot frontal plane motion and peak KAMs, indicating that increased forefoot inversion was related to reduced knee impulse and KAM during mid and late stance phase. Due to the coupling movement between the rearfoot and forefoot [38], inversion of the forefoot during stance phase is affected by the degree of rearfoot eversion, therefore the greater rearfoot eversion in the knee OA group would make the forefoot relatively inverted. It is also possible that motion of the midfoot in the frontal plane affects medial knee loading, however due to the absence of a midfoot segment in the Oxford foot model, we are unable to determine the contribution of midfoot motion to altered medial knee loading. Kinematic foot models which allow for more detailed analysis of the midfoot may be of additional benefit to better understand foot function in people with medial compartment knee OA. Nevertheless, based on our current findings, the correlations found between frontal plane rearfoot and forefoot motion and peak KAMs suggest that those with greater peak rearfoot eversion and forefoot inversion exhibited reduced medial knee joint loading during the mid- to late stance phase of gait.

Interestingly, the associations found between foot kinematics and KAMs did not involve the $1^{\text {st }}$ peak KAM, which is most often targeted with load-altering interventions. There are a number of possible explanations for this. Firstly, there are differences in timing of when these peaks occur during gait. The $1^{\text {st }}$ peak KAM for the knee OA group occurred at early stance (average 19.1\% gait cycle) while peak rearfoot eversion occurred at $30 \%$ of the gait cycle. Secondly, it is possible that the movement of the midfoot may be related to medial knee loading, as midfoot motion can compensate for rearfoot motion. Further investigation, however, is required to ascertain this. Lastly, previous studies have suggested that there may be distinct subgroups of individuals based on timing patterns of rearfoot frontal plane motion [39]. Participants with an "early" rearfoot eversion pattern (ie. rapid eversion in the first $10 \%$ gait cycle) may be more likely to exhibit changes in KAM associated with load-altering interventions, which may explain why these interventions are more effective in some subpopulations than others. It is also important to acknowledge that KAAI has been shown to be a more sensitive mechanical joint loading parameter than peak KAMs [40]. Given that the KAAI takes into account both the magnitude and duration of knee medial loading, the correlation between greater peak rearfoot eversion and forefoot inversion and reduced KAAI may indicate overall reduction of medial knee joint loading during stance.

Reports of the biomechanical effects of different load modifying interventions (orthotics and shoes) have been inconsistent [10,25,32,33]. While some studies investigating lateral wedged insoles have reported a reduction in the KAMs $[10,11,33,41]$, others have reported an increase in KAM $[25,32,33]$. Different insole lengths have also shown different responses, with full length insoles being more effective at reducing KAM than heel wedges [10]. These findings support the suggestion that there may be sub-groups that better respond to lateral wedged insoles, and that variability in response to orthotic intervention may be evident in people with medial compartment knee OA [24]. Footwear-related interventions, such as variable stiffness shoes, have also demonstrated a reduction in the $\operatorname{KAM}[6,12,42]$ with evidence suggesting a reduction in the medial compartment in vivo contact force [12]. It may be possible that insole interventions which aim at modifying motion of the whole foot (rearfoot, midfoot and forefoot), such as shoes and full length insoles, may be more effective due to their effect on forefoot frontal plane motion in addition to altering rearfoot motion. However, due to the potential high variability in response to load modifying interventions, appropriate individual screening of the lower limb may need to be undertaken to assess the suitability of the intervention and to achieve optimal clinical outcomes.

\section{Conclusion}

Associations between kinematic measures at the foot and moments at the knee indicate that increased rearfoot eversion, rearfoot internal rotation and forefoot inversion are associated with reduced KAM and KAAI during the stance phase of gait. These findings suggest that medial knee joint loading is reduced in people with OA who walk with greater foot pronation. Due to the high variability reported to load modifying interventions in people with medial compartment knee OA, individual screening of the lower limb may need to be performed to assess suitability for these interventions and to achieve optimal clinical outcomes. 


\section{Abbreviations}

OA: Osteoarthritis; KAM: Knee adduction moment; KAAl: Knee adduction angular impulse.

\section{Competing interests}

HBM is Editor-in-Chief of the Journal of Foot and Ankle Research. It is journal policy that editors are removed from the peer review and editorial decision making processes for papers they have co-authored.

\section{Authors' contributions}

PL: designed and managed the study, collected and analysed the data drafted the manuscript. HBM: participated in the study design and assisted in the statistical analysis and data interpretation, helped to draft the manuscript. ADM: assisted in data collection, data analysis. JF, JB and NB have assisted in patient recruitment, grading $x$-ray severity and drafting the manuscript. PL, HBM and JF obtained the funding. All authors have read and approved the final version.

\section{Acknowledgements}

This study was funded by the Clive and Vera Ramaciotti Foundation and the Arthritis Foundation of Australia. HBM is currently a National Health and Medical Research Council Senior Research Fellow (ID: 1020925). PL is currently funded through the Australian Government's Collaborative Research Networks programme.

\section{Author details}

${ }^{1}$ Institute of Sport, Exercise and Active Living, College of Sport and Exercise Science, Victoria University, Melbourne Vic 8001, Australia. ${ }^{2}$ Lower Extremity and Gait Studies Program, Faculty of Health Sciences, La Trobe University, Melbourne Vic 3086, Australia. ${ }^{3}$ Warringal Medical Centre, Melbourne Vic 3084, Australia.

Received: 18 April 2013 Accepted: 25 June 2013

Published: 8 August 2013

\section{References}

1. Access Economics Report: Painful realities: the economic impact of arthritis in Australia in 2007. Arthritis Australia; 2007

2. Woolf $A D$, Pfleger $B$ : Burden of major musculoskeletal conditions. Bull World Health Organ 2003, 81:646-656.

3. Andriacchi TP: Dynamics of knee malalignment. Orthop Clin North Am 1994, 25:395-403.

4. Mills K, Blanch P, Chapman AR, McPoil TG, Vicenzino B: Foot orthoses and gait: a systematic review and meta-analysis of literature pertaining to potential mechanisms. Br J Sports Med 2010, 44:1035-1046.

5. Foroughi N, Smith R, Vanwanseele B: The association of external knee adduction moment with biomechanical variables in osteoarthritis: a systematic review. Knee 2009, 16:303-309.

6. Erhart JC, Mundermann A, Elspas B, Giori NJ, Andriacchi TP: A variablestiffness shoe lowers the knee adduction moment in subjects with symptoms of medial compartment knee osteoarthritis. J Biomech 2008, 41:2720-2725

7. Shakoor N, Lidtke RH, Sengupta M, Fogg LF, Block JA: Effects of specialized footwear on joint loads in osteoarthritis of the knee. Arthritis Rheum 2008, 59:1214-1220.

8. Shelburne KB, Torry MR, Steadman JR, Pandy MG: Effects of foot orthoses and valgus bracing on the knee adduction moment and medial joint load during gait. Clin Biomech (Bristol, Avon) 2008, 23:814-821.

9. Baker K, Goggins J, Xie H, Szumowski K, LaValley M, Hunter DJ, Felson DT: A randomized crossover trial of a wedged insole for treatment of knee osteoarthritis. Arthritis Rheum 2007, 56:1198-1203.

10. Hinman RS, Bowles KA, Payne C, Bennell KL: Effect of length on laterallywedged insoles in knee osteoarthritis. Arthritis Rheum 2008, 59:144-147.

11. Hinman RS, Payne C, Metcalf BR, Wrigley TV, Bennell KL: Lateral wedges in knee osteoarthritis: what are their immediate clinical and biomechanical effects and can these predict a three-month clinical outcome? Arthritis Rheum 2008, 59:408-415.

12. Erhart JC, Dyrby CO, D'Lima DD, Colwell CW, Andriacchi TP: Changes in in vivo knee loading with a variable-stiffness intervention shoe correlate with changes in the knee adduction moment. J Orthop Res 2010, 28:1548-1553.
13. Teichtahl AJ, Morris ME, Wluka AE, Baker R, Wolfe R, Davis SR, Cicuttini FM: Foot rotation-a potential target to modify the knee adduction moment. J Sci Med Sport 2006, 9:67-71.

14. Miyazaki T, Wada M, Kawahara H, Baba H, Shimada S: Dynamic load at baseline can predict radiographic disease progression in medial compartment knee. Ann Rheum Dis 2002, 61:617-622.

15. Lin C, Lai $K$, Chou Y, Ho C: The effect of changing foot progression angle on the knee adduction moment in normal teenagers. Gait Posture 2001, 14:85-91.

16. Wada M, Maezawa Y, Baba H, Shimada S, Sasaki S, Nose Y: Relationships among bone mineral densities, static alignment and dynamic load in patients with medial compartment knee osteoarthritis. Rheumatol 2001, 40:499-505

17. Guo M, Axe MJ, Manal K: The influence of foot progression angle on the knee adduction moment during walking and stair climbing in pain free individuals with knee osteoarthritis. Gait Posture 2007, 26:436-441.

18. Felson DT, Goggins J, Niu J, Zhang Y, Hunter DJ: The effect of body weight on progression of knee osteoarthritis is dependent on alignment. Arthritis Rheum 2004, 50:3904-3909.

19. McWilliams DF, Doherty S, Maciewicz RA, Muir KR, Zhang W, Doherty M: Self-reported knee and foot alignments in early adult life and risk of osteoarthritis. Arthritis Care Res (Hoboken) 2010, 62:489-495.

20. Sharma L, Song J, Dunlop D, Felson D, Lewis CE, Segal N, Torner J, Cooke TD, Hietpas J, Lynch J, Nevitt M: Varus and valgus alignment and incident and progressive knee osteoarthritis. Ann Rheum Dis 2010, 69:1940-1945.

21. Levinger P, Menz HB, Fotoohabadi MR, Feller JA, Bartlett JR, Bergman NR Foot posture in people with medial compartment knee osteoarthritis J Foot Ankle Res 2010, 3:29.

22. Reilly K, Barker K, Shamley D, Newman M, Oskrochi GR, Sandall S: The role of foot and ankle assessment of patients with lower limb osteoarthritis. Physiotherapy 2009, 95:164-169.

23. Reilly K, Barker K, Shamley D, Sandall S: Influence of foot characteristics on the site of lower limb osteoarthritis. Foot Ankle Int 2006, 27:206-211.

24. Levinger P, Menz HB, Morrow AD, Feller JA, Bartlett JR, Bergman NR: Foot kinematics in people with medial compartment knee osteoarthritis. Rheumatol 2012, 51:2191-2198.

25. Kakihana W, Akai M, Nakazawa K, Takashima T, Naito K, Torii S: Effects of laterally wedged insoles on knee and subtalar joint moments. Arch Phys Med Rehabil 2005, 86:1465-1471.

26. Chiba H, Ebihara S, Tomita N, Sasaki H, Butler JP: Differential gait kinematics between fallers and non-fallers in community dwelling elderly people. Geriatr Gerontol Int 2005, 5:127-134.

27. Brandt K, Fife R, Braunstein E: Radiographic grading of the severity of knee osteoarthritis: relation of the Kellgren and Lawrence grade to a grade based on joint space narrowing and correlation with arthroscopic evidence of articular cartilage degeneration. Arthritis Rheum 1989, 32:1584-1591.

28. Kadaba MP, Ramakrishnan HK, Wootten ME: Measurement of lower extremity kinematics during level walking. J Orthop Res 1990, 8:383-392.

29. Stebbins J, Harrington M, Thompson N, Zavatsky A, Theologis T: Repeatability of a model for measuring multi-segment foot kinematics in children. Gait Posture 2006, 23:401-410.

30. Thorp LE, Sumner DR, Block JA, Moisio KC, Shott S, Wimmer MA: Knee joint loading differs in individuals with mild compared with moderate medial knee osteoarthritis. Arthritis Rheum 2006, 54:3842-3849.

31. Bellamy N: Pain assessment in osteoarthritis: experience with the WOMAC osteoarthritis index. Semin Arthritis Rheum 1989, 18:14-17.

32. Kakihana W, Akai M, Nakazawa K, Naito K, Torii S: Inconsistent knee varus moment reduction caused by a lateral wedge in knee osteoarthritis. Am J Phys Med Rehabil 2007, 86:446-454.

33. Hinman RS, Bowles KA, Metcalf BB, Wrigley TV, Bennell KL: Lateral wedge insoles for medial knee osteoarthritis: effects on lower limb frontal plane biomechanics. Clin Biomech 2012, 27:27-33.

34. Yagi T, Sasaki T: Tibial torsion in patients with medial-type osteoarthritic knee. Clin Orthop Relat Res 1986:177-182.

35. Levinger P, Menz HB, Morrow AD, Bartlett JR, Feller JA, Fotoohabadi MR, Bergman NR: Dynamic foot function changes following total knee replacement surgery. Knee 2012, 19:880-885.

36. Lindgren $U$, Seireg A: The influence of mediolateral deformity, tibial torsion, and foot position on femorotibial load. Prediction of a musculoskeletal computer model. Arch Orthop Trauma Surg 1989, 108:22-26. 
37. Levinger P, Murley GS, Barton CJ, Cotchett MP, McSweeney SR, Menz HB: A comparison of foot kinematics in people with normal- and flat-arched feet using the Oxford Foot Model. Gait Posture 2010, 32:519-523.

38. Cornwall MW, McPoil TG: Motion of the calcaneus, navicular, and first metatarsal during the stance phase of walking. J Am Podiatr Med Assoc 2002, 92:67-76.

39. Cornwall MW, MCPoil TG: Classification of frontal plane rearfoot motion patterns during the stance phase of walking. I Am Podiatr Med Assoc 2009, 99:399-405.

40. Kean CO, Hinman RS, Bowles KA, Cicuttini F, Davies-Tuck M, Bennell KL: Comparison of peak knee adduction moment and knee adduction moment impulse in distinguishing between severities of knee osteoarthritis. Clin Biomech 2012, 27:520-523.

41. Butler RJ, Marchesi S, Royer T, Davis IS: The effect of a subject-specific amount of lateral wedge on knee mechanics in patients with medial knee osteoarthritis. J Orthop Res 2007, 25:1121-1127.

42. Erhart JC, Mundermann A, Elspas B, Giori NJ, Andriacchi TP: Changes in knee adduction moment, pain, and functionality with a variable-stiffness walking shoe after 6 months. J Orthop Res 2010, 28:873-879.

doi:10.1186/1757-1146-6-33

Cite this article as: Levinger et al:: Relationship between foot function and medial knee joint loading in people with medial compartment knee osteoarthritis. Journal of Foot and Ankle Research 2013 6:33.

\section{Submit your next manuscript to BioMed Central and take full advantage of:}

- Convenient online submission

- Thorough peer review

- No space constraints or color figure charges

- Immediate publication on acceptance

- Inclusion in PubMed, CAS, Scopus and Google Scholar

- Research which is freely available for redistribution 